\title{
Evidence for oesophageal and anorectal involvement in very early systemic sclerosis (VEDOSS): report from a single VEDOSS/EUSTAR centre
}

\author{
Gemma Lepri, ${ }^{1}$ Serena Guiducci, ${ }^{1}$ Silvia Bellando-Randone, ${ }^{1}$ Iacopo Giani, ${ }^{2}$ \\ Cosimo Bruni, ${ }^{1}$ Jelena Blagojevic, ${ }^{1}$ Giulia Carnesecchi, ${ }^{1}$ Alessandra Radicati, ${ }^{1}$ \\ Filippo Pucciani, ${ }^{3}$ Matucci-Cerinic Marco ${ }^{1}$
}

\section{Handling editor Tore K Kvien \\ ${ }^{1}$ Department of Biomedicine, Division of Rheumatology AOUC and Department of Clinical and Experimental Medicine, University of Florence, Florence, Italy ${ }^{2}$ General Surgery, USL-8 Arezzo, Arezzo, Italy ${ }^{3}$ Department of Surgery and Translational Medicine, University of Florence, Florence, Italy}

\section{Correspondence to} Professor Matucci-Cerinic Marco, Division of Rheumatology AOUC, Denothe Centre, Department of Biomedicine, University of Florence, Villa Monna Tessa, Viale Pieraccini 18 , Florence 50139, Italy; cerinic@unifi.it

Received 5 May 2013 Revised 7 August 2013 Accepted 19 September 2013 Published Online First 14 October 2013

\section{CrossMark}

To cite: Lepri G, Guiducci S, Bellando-Randone $S$, et al. Ann Rheum Dis

2015;74:124-128.

\section{ABSTRACT}

Background The oesophagus is the first gastrointestinal (GI) tract involved in systemic sclerosis (SSc), followed by the anorectum.

Objective Evaluation of oesophageal and anorectal involvement and their correlations in patients with very early diagnosis of SSC (VEDOSS).

Patients and methods 59 patients with VEDOSS, evaluated with oesophageal and anorectal manometry and investigated with lung function tests and chest HRCT. Demographic data, oesophageal and anorectal symptoms, Raynaud's phenomenon, autoantibodies, videocapillaroscopy patterns, puffy fingers and digital ulcers were recorded for all patients.

Results In 4 patients oesophageal manometry and in 17 patients anorectal manometry was not performed because of scarce tolerance. Oesophageal peristalsis was absent in 14 patients; its pressure and speed were significantly lower in 41 patients $(p<0.001$ and $p=0.005$, respectively). The maximum pressure and mean pressure (Pmax and $\mathrm{Pm}$ ) of lower oesophageal sphincter were significantly lower $(p=0.012$ and $p=0.024$, respectively). Patients with a diffusing capacity of the lung for carbon monoxide $<80 \%$ presented a hypotonic lower oesophageal sphincter $(p=0.008)$ and an abnormal peristalsis $(p<0.001)$; patients with a diffusing capacity of the lung for carbon monoxide $>80 \%$ showed only an abnormal peristalsis $(<0.001)$. The anal resting pressure (ARP) at $4.3 \mathrm{~cm}$ and $2 \mathrm{~cm}$ from anal edge and the anal canal Pm were significantly decreased $(p<0.001$ and $p=0.010$, respectively). The maximum voluntary contraction was significantly abnormal in its Pmax and $\mathrm{Pm}(p=0.017$ and $p=0.005)$ and in its duration $(p=0.001)$. In patients with a positive HRCT, the ARP and the canal Pmax and Pm were significantly lower; patients with negative HRCT presented only an abnormal ARP.

Conclusions In patients with VEDOSS, oesophageal and anorectal disorders are frequently detected, showing that very early SSc is characterised by $\mathrm{Gl}$ involvement.

\section{INTRODUCTION}

Systemic sclerosis (SSc) is characterised by the involvement of the gastrointestinal (GI) system in more than $80 \%$ of the patients. ${ }^{1}$ The oesophagus is involved with a prevalence of 50-90\% with a replacement of smooth muscle by collagenous fibrosis and smooth muscle atrophy leading to motor activity abnormalities. ${ }^{2}$ The distal two-thirds and the lower oesophageal sphincter (LOS), composed of visceral smooth muscles, are the main target of $\mathrm{SSc}^{3}{ }^{4}$ with a decreased pressure or total LOS incompetence, and distal oesophageal peristaltic waves abnormalities. ${ }^{3}$ 4-6 This motor dysfunction leads to gastro-oesophageal reflux disease which may be clinically asymptomatic or characterised by heartburn, regurgitation and dysphagia (leading to stenosis and Barrett's oesophagus with a higher risk of oesophageal cancer). ${ }^{3}$ In SSc, the anorectum is the second most studied area of the GI tract $^{6}$ and its dysfunction is reported with a prevalence of $50-70 \%$ with a high impact on patients' quality of life. ${ }^{67}$ The internal anal sphincter (IAS), is a smooth muscle and at rest has a tonic contraction responsible for $85 \%$ of the continence of the anal canal. In SSc, the majority of studies show that the resting tone of anal sphincter, composed primarily by the IAS, is decreased ${ }^{5}{ }^{89}$ and that the IAS impaired function is the main mechanism of faecal incontinence. ${ }^{1} \quad 3 \quad 4 \quad 10$ The rectoanal inhibitory reflex (RAIR) is reduced or absent in $12-80 \%$ of patients with SSc. ${ }^{2}{ }^{5}$ A decrease of maximum contraction pressure and of rectal compliance and capacity is also frequent. ${ }^{5} 811$ Patients with SSc with anorectal involvement may present with constipation, diarrhoea, increased rectal fullness or urgency and incontinence. ${ }^{5}$ However, patients may be also asymptomatic or embarrassed to report these symptoms. ${ }^{58}$

Recently EUSTAR (European League Against Rheumatism Scleroderma Trial and Research Group) has proposed new criteria for a very early diagnosis of SSc (VEDOSS) that have yet to be validated and that are represented by the presence of the three red flags (Raynaud's phenomenon, puffy fingers and antinuclear antibodies (ANAs) positivity) plus disease-specific autoantibodies (anticentromere $\mathrm{Ab}(\mathrm{ACA})$ or anti-topo I Ab (Scl70)) or microvascular alterations detected by nailfold videocapillaroscopy. VEDOSS may allow to identify the phase before the skin becomes involved and the disease is classified as definite. ${ }^{12} 13$

The aim of this study was to evaluate the presence of oesophageal and anorectal involvement in patients with VEDOSS, subjecting these patients to oesophageal and anorectal manometry that represents a validated diagnostic tool to detect GI 
dysmotility. ${ }^{5}$ However it is important to remark the promising role of impedance to assess the bolus transit and gastrooesophageal reflux. ${ }^{14}$

\section{PATIENTS AND METHODS}

From May 2010 to April 2012, 59 patients (58 women) followed at the Division of Rheumatology of the University of Florence and participating to a prospective database (EUSTAR, VEDOSS-Project, http://www.eustar.org), met the VEDOSS criteria $^{13}$ and were included in the study. Exclusion criteria were: age $>80$ years or $<18$ years, impaired general health, neurological disease, physical handicap, previous oesophageal and/or anorectal surgery. All patients received a preliminary clinical evaluation and were studied by mean oesophageal and anorectal manometry at the Surgery Clinic of the University of Florence. Their results were compared with clinical and manometric data previously obtained from control subjects who did not present with GI problems and were submitted to minor day surgery; respectively 24 subjects (19 women) were investigated with oesophageal manometry and 21 (12 women) with anorectal manometry. These subjects have been evaluated by the same surgeon in the same clinic.

Ethics Committee approval was obtained according to ethical guidelines of our university; all participants provided written consent to participate in the study with full knowledge of the procedure.

Before manometric evaluation, the following GI symptoms have been investigated and registered: dysphagia, typical gastrooesophageal reflux disease symptoms (as heartburn or regurgitation), urgency and/or incontinence, constipation and diarrhoea.

The demographic data, resting pressure (RP, presence/absence, duration), autoantibody profile (ANA, ACA, topo-I), videocapillaroscopic patterns (Normal, Early, Active, Late), ${ }^{15}$ puffy fingers, digital ulcers (DUs), chest high resolution computed tomography (HRCT), pulmonary function tests (PFTs) with diffusing capacity of the lung for carbon monoxide (DLCO) were recorded for all patients.

\section{Oesophageal manometry}

All drugs affecting the contractile oesophageal patterns (calcium channel blockers, $\beta$-blockers and nitrates) were discontinued 1 week before examination and all patients underwent a X-ray evaluation of the oesophagus in advance to exclude organic diseases. Oesophageal manometry was performed after $12 \mathrm{~h}$ of fasting by the same physician with an 8-channel catheter (EUCAT, Medimar s.r.l., Milan) perfused at $0.5 \mathrm{~mL} / \mathrm{min}$ by a lowcompliance perfusion pump. ${ }^{16} 17$ After the catheter was placed with the distal recording site located in the stomach, it was slowly withdrawn three times (slow pull-throughs). The three pull-throughs identified the high pressure zone of the LOS-RP. Successively, postdeglutitive LOS relaxation and oesophageal peristalsis were evaluated. Finally the catheter was withdrawn to detect the high pressure zone of the upper oesophageal sphincter (UOS-RP). ${ }^{17}$ Recordings and analyses of the tracings were made using a computerised system (Dyno System, Menfis bioMedica s. r.l. Bologna). Computerised analysis identified the maximal pressure (Pmax) and the mean pressure $(\mathrm{Pm})$ among the LOS-RPs and UOS-RPs. The maximum pressure of peristaltic waves (Peristalsis Pmax), the mean pressure (Peristalsis Pm) and the speed of peristalsis (Peristalsis speed) were reported.

\section{Anorectal manometry}

All anorectal manometries were performed by the same physician. Patients were examined after two enemas (one the day prior to the examination and the second $2 \mathrm{~h}$ prior to the manometry): previously, all patients performed a colonoscopy to exclude organic diseases.

Anorectal manometry, with the patient in the left lateral position with knees and hips bent to $90^{\circ}$, was carried out with an 8-channel water-perfused flexible catheter (ISCHIA, Menfis bioMedica s.r.l. Bologna). A low compliance pneumohydraulic infusion pump was used to maintain catheter perfusion at $0.5 \mathrm{~mL} / \mathrm{min}$.

The lubricated manometry probe was gently inserted into the rectum and oriented such that the most distal sensor $11 \mathrm{~cm}$ level) is located posteriorly at $1 \mathrm{~cm}$ from anal verge. ${ }^{9} 18$ The recordings and the analyses of the tracings were made by a computerised system (Dyno Compact, Menfis bioMedica s.r.l., Bologna). Among the anal RPs (ARPs) computerised analysis identified the maximal anal pressure (Pmax) and the mean pressure $(\mathrm{Pm})$ of the anal canal. The maximal voluntary contraction (MVC) was evaluated by asking the subject to voluntarily contract the anal sphincter as long as possible. The computer quantified the amplitude in $\mathrm{mmHg}$ and duration in seconds. The RAIR was elicited by inflating a soft rubber balloon in the anorectum at $10 \mathrm{~cm}$ from the anal verge: the volume was increased every $20 \mathrm{~mL}$. In all patients the first distension volume at which internal sphincter relaxation occurred (RAIR threshold) and the distension volume for which an initial transient sensation occurred (conscious rectal sensitivity threshold, CRST) were determined. The maximal tolerated volume (MTV) was also measured in all patients; it was considered an expression of rectal reservoir capacity. Compliance of the rectum (expression of the ratio $\mathrm{mmHg} / \mathrm{mL}$ of inflated air) was detected by means of the pressure/volume curve. The manometric procedure ended by measuring anal pressures during attempted defaecation (straining test). The straining test was considered positive if inappropriate rise or less than $20 \%$ relaxation of basal RP occurred. $^{18}$

\section{Statistical analysis}

Data are given as mean \pm SD and median. Data were evaluated by Mann-Whitney U test, a non-parametric statistical test; a $\mathrm{p}$ value of $<0.05$ was considered statistically significant.

All correlations were evaluated using the Spearman's rank correlation coefficient $\left(\rho_{s}\right)$ where full correlation is 1 and correlation $<0.50$ is considered not significant.

\section{RESULTS}

In 4 patients, oesophageal manometry was not performed because of scarce tolerance and in 17 patients anorectal manometry was not made for general problems (distance from the outpatient unit, non-collaboration). Clinical and serological features of the patients are shown in table 1 . Twenty-seven of 55 (49.1\%) patients evaluated with oesophageal manometry and $6 / 42(14.3 \%)$ evaluated with anorectal manometry were symptomatic. Results of oesophageal and anorectal studies are shown in tables 2 and 3. All patients were not smokers and haemoglobin was normal.

\section{Oesophageal manometry}

Peristalsis was absent in $14 / 55$ patients $(25.5 \%)$. In patients with VEDOSS, the peristalsis Pmax and $\operatorname{Pm}(\mathrm{p}<0.001)$ and the peristalsis speed $(\mathrm{p}=0.005)$ were significantly lower when compared with controls. Similarly the LOS Pmax and Pm were significantly lower ( $p=0.012$ and 0.024 , respectively), showing these patients to have a hypotonic LOS when compared with controls. 


\begin{tabular}{|c|c|c|}
\hline Data & $\begin{array}{l}\text { Patients evaluated } \\
\text { with oesophageal } \\
\text { manometry (n: } 55 \text { ) }\end{array}$ & $\begin{array}{l}\text { Patients evaluated } \\
\text { with anorectal } \\
\text { manometry (n: 42) }\end{array}$ \\
\hline Age (years) & $48.5 \pm 14.2$ & $51.7 \pm 12.5$ \\
\hline $\begin{array}{l}\text { Duration of Raynaud's } \\
\text { phenomenon (years) }\end{array}$ & $6.6 \pm 7.3$ & $6 \pm 6.5$ \\
\hline Puffy fingers & $\mathrm{n}=21 / 55(38.2 \%)$ & $n=14 / 42(33.3 \%)$ \\
\hline mRSS & 0 & 0 \\
\hline \multicolumn{3}{|l|}{ Autoantibodies: } \\
\hline Only ANA & $n=20 / 55(36.4 \%)$ & $n=13 / 42(30.9 \%)$ \\
\hline $\mathrm{ANA}+\mathrm{ACA}$ & $\mathrm{n}=24 / 55(43.6 \%)$ & $\mathrm{n}=21 / 42(50 \%)$ \\
\hline $\mathrm{ANA}+\mathrm{Scl} 70$ & $n=11 / 55(20 \%)$ & $\mathrm{n}=8 / 42(19 \%)$ \\
\hline Digital ulcers & $n=13 / 55(23.6 \%)$ & $n=10 / 42(23.8 \%)$ \\
\hline \multicolumn{3}{|l|}{ Capillaroscopy } \\
\hline Aspecific pattern & $n=14 / 55(25.5 \%)$ & $\mathrm{n}=11 / 42(26.2 \%)$ \\
\hline Early scleroderma pattern & $n=27 / 55(49.1 \%)$ & $n=19 / 42(45.2 \%)$ \\
\hline Active scleroderma pattern & $\mathrm{n}=11 / 55(20 \%)$ & $n=10 / 42(23.8 \%)$ \\
\hline Late scleroderma pattern & $\mathrm{n}=3 / 55(5.5 \%)$ & $\mathrm{n}=2 / 42(4.8 \%)$ \\
\hline Oesophageal symptoms & $\mathrm{n}=27 / 55(49.1 \%)$ & $\mathrm{n}=2 / 42(4.8 \%)$ \\
\hline
\end{tabular}

ACA, anticentromere Ab; ANA, antinuclear antibody; mRSS, modified Rodnan Skin Score.

Oesophageal manometric parameters (LOSP and oesophageal motility) did not correlate with autoantibodies, duration of RP, DU, puffy fingers $\left(\rho_{\mathrm{s}}>0.05\right)$.

In patients without oesophageal symptoms, peristalsis speed was significantly lower $(\mathrm{p}=0.012)$ when compared with symptomatic patients.

However, out of 14 patients without peristaltic waves 12 presented with oesophageal symptoms.

LOS pressure was significantly abnormal in patients with early $(p=0.026)$, active $(p=0.009)$ and late $(p=0.007)$ pattern at videocapillaroscopy when compared with controls.

It is interesting to remark that patients with aspecific $(\mathrm{p}<0.001)$, early $(\mathrm{p}<0.001)$ and active $(\mathrm{p}<0.001$ and $\mathrm{p}=0.002$ ) capillaroscopic patterns presented peristalsis (Pmax and Pm) abnormalities.

\section{Oesophageal manometry and lung involvement}

Fifty-one patients with VEDOSS were investigated with PFTs and $25 / 51$ presented a DLCO $<80 \%$ (a detection of DLCO $<80 \%$ was considered indicative of an early modification of lung function ${ }^{19}$ ) while forced vital capacity was always found normal.

There were no significant differences in LOS pressure and UOS pressure between the two populations with VEDOSS with DLCO $>80 \%$ and with DLCO $<80 \%$. However, statistically significant differences between the two subgroups were observed when compared with controls: in fact, patients with DLCO $>80 \%$ did not present statistically significant differences in LOS pressure while those with DLCO $<80$ showed a statistically significant difference in LOS Pmax and in the LOS Pm (respectively $\mathrm{p}=0.008$ and $\mathrm{p}=0.008$ ) when compared with controls.

There were no significant differences in peristalsis between the two subgroups of patients with VEDOSS (DLCO $>80 \%$ and DLCO $<80 \%$ ), and also patients with DLCO $>80 \%$ showed an abnormal peristalsis, as peristalsis speed, peristalsis Pmax and its $\mathrm{Pm}$ were all significantly lower in population with DLCO $>80 \%$ $(\mathrm{p}=0.005, \mathrm{p}<0.001$ and $\mathrm{p}<0.001$, respectively). Thus patients with DLCO $>80 \%$ already presented with abnormal peristalsis when compared with controls. Moreover 8/26 patients $(30.8 \%)$ did not have peristalsis.

Comparing patients with DLCO $<80 \%$ to controls, the peristalsis Pmax and its Pm were significantly lower $(\mathrm{p}<0.001)$.

Out of 55 patients investigated with oesophageal manometry, 45 were also investigated with chest HRCT: $23 / 45$ presented a negative HRCT, while $22 / 45$ presented a lung involvement (interlobular and intralobular septa thickening, areas of fibrotic thickening, areas of ground glass). In both groups a significantly abnormal peristalsis was detected $(\mathrm{p}<0.001)$. In $8 / 23$ patients with negative chest HRCT (34.8\%) and in $4 / 22$ patients with positive HRCT (18.2\%), peristalsis was absent.

\section{Anorectal manometry}

When compared with controls, patients with VEDOSS had the ARP at $4 \mathrm{~cm}, 3 \mathrm{~cm}$ and $2 \mathrm{~cm}$ from anal edge significantly lower $(\mathrm{p}<0.001)$ and the anal canal Pm significantly abnormal $(p=0.010)$. Comparing our patients with controls also the MVC was significantly abnormal as its Pmax, Pm and duration (duration and duration 50\%) were significantly lower $(\mathrm{p}=0.017, \mathrm{p}=0.005, \mathrm{p}=0.01$ and $\mathrm{p}=0.03$, respectively).

The CRST, the constant sensation and the MTV were normal.

Anorectal manometric parameters did not correlate with autoantibodies, duration of RP, DU and puffy fingers $\left(\rho_{s}>0.05\right)$.

Patients with symptoms of anorectal involvement presented a significant lower anal canal Pmax and Pm (respectively $\mathrm{p}=0.19$ and $\mathrm{p}=0.039$ ) when compared with asymptomatic subjects.

Patients with an aspecific pattern at videocapillaroscopy and patients with a scleroderma pattern (early, active, late) presented a significantly abnormal ARP at $4 \mathrm{~cm}, 3 \mathrm{~cm}$ and $2 \mathrm{~cm}$ from anal edge $(\mathrm{p}<0.001)$.

\section{Anorectal manometry and lung involvement}

Out of 42 patients investigated with anorectal manometry 40 were investigated with PFTs and 21 presented with a DLCO $<80 \%$.

There were no statistically significant differences in anorectal manometry between patients with DLCO $<80 \%$ and patients with DLCO $>80 \%(p>0.05)$. Both populations presented a significantly lower ARP $(\mathrm{p}<0.001)$ when compared with controls. However, in patients with a DLCO $>80 \%$, a significantly lower anal canal Pm $(p=0.001)$ was also detected. Patients with a DLCO $<80 \%$ showed an abnormal MVC Pmax and Pm $(\mathrm{p}=0.019$ and $\mathrm{p}=0.012$, respectively) and duration (duration and duration50\%) $(\mathrm{p}<0.001$ and $\mathrm{p}=0.001$, respectively), instead in patients with DLCO $>80 \%$ only an abnormal MVC Pm and MVC duration50\% were detected $(\mathrm{p}=0.028$ and $\mathrm{p}=0.025$, respectively).

Out of 42 patients investigated with anorectal manometry, 35 were investigated with chest HRCT: $17 / 35$ presented with a negative HRCT, while $18 / 45$ presented with a lung involvement.

The groups of patients with positive HRCT and negative HRCT presented with an abnormal ARP at $4 \mathrm{~cm}, 3 \mathrm{~cm}$ and $2 \mathrm{~cm}$ from anal edge $(\mathrm{p}<0.001)$ when compared with controls. Patients with positive HRCT even presented with a significant decrease of anal canal Pmax and $\operatorname{Pm}(p=0.010$ and $p<0.001$, respectively) if compared with controls.

Moreover, we found that patients with a positive HRCT presented with a significant alteration of MVC Pmax, Pm, duration and duration 50\% $\quad(\mathrm{p}=0.025, \mathrm{p}=0.008 ; \mathrm{p}<0.001$ and $\mathrm{p}=0.001$, respectively) if compared with controls. Patients with negative HRCT presented with only a significant alteration of MVC duration $(\mathrm{p}=0.017)$. 
Table 2 Results of oesophageal manometry

\begin{tabular}{|c|c|c|c|c|c|c|c|}
\hline \multirow[b]{2}{*}{ Parameter } & \multicolumn{3}{|c|}{ Patients with very early diagnosis of SSC } & \multicolumn{3}{|l|}{ Controls } & \multirow[b]{2}{*}{ p Value } \\
\hline & Number of patients & Mean value \pm SD & Median & Number of patients & Mean value \pm SD & Median & \\
\hline LOS Pmax (mmHg) & 55 & $34.54 \pm 16.06$ & 33 & 24 & $40.45 \pm 7.89$ & 40.30 & 0.012 \\
\hline LOS Pm (mmHg) & 55 & $10.21 \pm 5.55$ & 9.10 & 24 & $11.51 \pm 2.66$ & 11.45 & 0.024 \\
\hline Peristalsis Speed (sec) & $41^{*}$ & $31.91 \pm 14.74$ & 30 & 24 & $36.85 \pm 10.45$ & 36.65 & 0.005 \\
\hline Peristalsis Pmax (mmHg) & $41^{*}$ & $43.47 \pm 20.09$ & 38.80 & 24 & $69.14 \pm 10.11$ & 69.40 & $<0.001$ \\
\hline Peristalsis Pm (mmHg) & $41^{*}$ & $20.24 \pm 9.96$ & 16.90 & 24 & $34.94 \pm 4.61$ & 34.80 & $<0.001$ \\
\hline Peristalsis duration (sec) & $41^{*}$ & $3.88 \pm 1.03$ & 3.70 & 24 & $3.50 \pm 0.37$ & 3.45 & 0.132 n.s. \\
\hline UOS Pmax (mmHg) & 55 & $90.86 \pm 58.10$ & 76.50 & 24 & $81.91 \pm 35.48$ & 75.45 & 0.881 n.s. \\
\hline UOS Pm(mmHg) & 55 & $35.79 \pm 24.45$ & 28.60 & 24 & $28.78 \pm 11.22$ & 25.85 & 0.519 n.s. \\
\hline
\end{tabular}

${ }^{*}$ Number of patients for whom it was possible to evaluate the manometric parameter (in 14/55 patients peristalsis was absent).

LOS, lower oesophageal sphincter; n.S., not significant; Pm, mean pressure; Pmax, maximum pressure; SSc, systemic sclerosis; UOS, upper oesophageal sphincter.

\section{DISCUSSION}

Our data show, for the first time, that in the majority of patients with VEDOSS an oesophageal and anal involvement is present and that in some of these patients lung involvement is also already detectable. This evidence suggests that these patients, presently classified very early, are not really in a very early phase of SSc, but they may be reasonably classified in the next early phase. ${ }^{20}$

We have found that in patients with VEDOSS, the LOS pressure and peristalsis were significantly abnormal, and oesophageal symptoms were detected in $49.1 \%$. However in patients without oesophageal symptoms, peristalsis speed is significantly lower if compared with symptomatic patients. Therefore it is clear that the absence of symptoms does not exclude an oesophageal involvement and that in asymptomatic patients GI involvement should also be always suspected, as in established SSc. ${ }^{34}$

It is important to remark that oesophageal involvement may also be found in patients without modification of lung function while a specific group of patients, likely representing a more advanced early phase in VEDOSS, already shows an oesophageal and lung involvement. This evidence in these patients without skin involvement and characterised by the main VEDOSS signs (RP, puffy fingers, ANA, antibodies/nailfold videocapillaroscopy (NVC)) may allow to identify three different subgroups: the first, without any organ involvement, the second with oesophageal/anal involvement and third with oesophageal/anal involvement and modification of lung function. For this reason, patients of the second and the third groups may not be considered anymore as VEDOSS but could be classified, as recently proposed, as early SSc. ${ }^{20}$ This evidence may indeed focus the attention of the clinician on the fact that skin involvement may not be the leading sign to assess the disease activity and severity in the very early phase of SSc.

Our study clearly shows that anorectal disorders are frequently detected in patients with VEDOSS. The fact that the majority of patients was asymptomatic should not prevent the investigation for a possible anorectal involvement. Anorectal manometry showed a significant abnormal ARP and this result agrees with previous studies that detected an IAS involvement (composed by smooth muscle). ${ }^{9}{ }^{11}$ This finding may reflect the impairment of LOS (composed by smooth muscle). However, our study also show an impaired MVC (a property of striated muscle of the external anal sphincter $(\mathrm{EAS})^{8}{ }^{21}$ ), likely reflecting a premature EAS fatigue, suggesting that the mechanisms regulating the EAS' function are involved with SSc. In our patients with VEDOSS, no significant differences in the CRST, the constant sensation and the MTV were found, suggesting that in these patients the faecal sensation and the rectal compliance are still maintained.

Table 3 Results of anorectal manometry

\begin{tabular}{|c|c|c|c|c|c|c|c|}
\hline \multirow[b]{2}{*}{ Parameter } & \multicolumn{3}{|c|}{ Patients with very early diagnosis of SSC } & \multicolumn{3}{|l|}{ Controls } & \multirow[b]{2}{*}{ p Value } \\
\hline & Number of patients & Mean value \pm SD & Median & Number of patients & Mean value \pm SD & Median & \\
\hline Anal canal RP $(4 \mathrm{~cm})(\mathrm{mmHg})$ & 42 & $3.32 \pm 5.46$ & 1.35 & 21 & $18.48 \pm 10.67$ & 19.60 & $<0.001$ \\
\hline Anal canal RP $(3 \mathrm{~cm})(\mathrm{mmHg})$ & 42 & $11.43 \pm 13.67$ & 6.55 & 21 & $43.70 \pm 9.82$ & 41.10 & $<0.001$ \\
\hline Anal canal RP $(2 \mathrm{~cm})(\mathrm{mmHg})$ & 42 & $28.98 \pm 23.18$ & 23.60 & 21 & $63.26 \pm 13.17$ & 60.80 & $<0.001$ \\
\hline Anal canal RP(1 cm) $(\mathrm{mmHg})$ & 42 & $50.24 \pm 22.85$ & 50.55 & 21 & $59.33 \pm 15.82$ & 59.60 & 0.067 n.s. \\
\hline Anal canal Pmax (mmHg) & 42 & $77.82 \pm 31.22$ & 80.40 & 21 & $86.49 \pm 19.14$ & 86.70 & 0.141 n.s. \\
\hline Anal canal $\mathrm{Pm}(\mathrm{mmHg})$ & 42 & $37.48 \pm 14.23$ & 38.35 & 21 & $46.15 \pm 6.42$ & 44.50 & 0.010 \\
\hline MVC Pmax (mmHg) & 42 & $71.69 \pm 39.01$ & 68.10 & 21 & $106.71 \pm 52.12$ & 108.50 & 0.017 \\
\hline MVC Pm (mmHg) & 42 & $44.59 \pm 23.25$ & 39.80 & 21 & $73.14 \pm 37.57$ & 76.10 & 0.005 \\
\hline MVC duration (sec) & 42 & $19.51 \pm 13.56$ & 15.25 & 21 & $32.83 \pm 12.15$ & 33.40 & 0.001 \\
\hline MVC duration $50 \%$ (sec) & $41^{*}$ & $12.88 \pm 12.65$ & 6.30 & 21 & $24.32 \pm 15.34$ & 20.40 & 0.003 \\
\hline CRST (mL) & 42 & $68.33 \pm 38.38$ & 60 & 21 & $51.90 \pm 9.81$ & 60 & 0.315 n.s. \\
\hline $\mathrm{CS}(\mathrm{mL})$ & 42 & $120 \pm 35.34$ & 100 & 21 & $108.09 \pm 10.78$ & 100 & 0.349 n.s. \\
\hline MTV (mL) & 42 & $192.86 \pm 36.65$ & 180 & 21 & $179.52 \pm 12.84$ & 180 & 0.229 n.s. \\
\hline
\end{tabular}

*Number of patients for whom it was possible to evaluate the manometric parameter (in one patient the MVC duration $50 \%$ not was evaluated).

CRST, conscious rectal sensitivity threshold; CS, constant sensation; MTV, maximal tolerated volume; MVC, maximal voluntary contraction; n.s., not significant; Pm, mean pressure; Pmax, maximum pressure. 
Moreover, anal symptoms (urgency/constipation) are correlated with a decreased Pmax and Pm of the anal canal. However, the absence of symptoms does not exclude an anorectal involvement (eg, an abnormal MVC pressure or duration).

As for oesophageal manometry it is important to highlight that patients without a modification of lung function may also present an early anorectal involvement. Thus patients with both DLCO $>80 \%$ and with DLCO $<80 \%$ presented with a significantly lower ARP. However, patients with DLCO $<80 \%$ showed a greater alteration of EAS.

In conclusion, our study demonstrates that patients with VEDOSS are characterised by oesophageal and anorectal involvement. In practice, this result suggests that these two GI tracts should always be investigated in patients with VEDOSS. In fact, clinical signs of organ impairment may appear later when the evolution to fibrosis is already underway while the disease has started several months or years before. From the pathophysiological point of view, Raynaud-associated neural dysfunction and immunological mechanisms could be the first step in the pathogenesis of GI involvement, followed by smooth muscle atrophy and then irreversible muscle fibrosis. ${ }^{22} \mathrm{~A}$ therapeutic strategy to avoid this evolution is still missing in patients with VEDOSS and further studies are warranted to provide more evidence to allow an early and aggressive treatment.

Correction notice This article has been corrected since it was published Online First. All authors' first and last names have been transposed.

Contributors M-CM conceived the study; LG conceived the study and participated in the drafting of the manuscript; PF performed manometries and participated in the drafting of the manuscript; GS, B-RS, GI, BC, BJ, CG and RA participated in the drafting of the manuscript.

Competing interests None.

Ethics approval Ethical Committee of AOU Careggi, University of Florence.

Provenance and peer review Not commissioned; externally peer reviewed.

\section{REFERENCES}

1 Savarino E, Mei F, Parodi A, et al. Gastrointestinal motility disorder assessment in systemic sclerosis. Rheumatology 2013;52:1095-100.

2 Marie I, Ducrotte $P$, Denis $P$, et al. Oesophageal mucosal involvement in patients with systemic sclerosis receiving proton pump inhibitor therapy. Aliment Pharmacol Ther 2006;24:1593-601.
3 Gyger G, Baron M. Gastrointestinal manifestations of scleroderma: recent progress in evaluation, pathogenesis, and management. Curr Rheumatol Rep 2012;14:22-9.

4 Domsic R, Fasanella K, Bielefeldt K. Gastrointestinal manifestations of systemic sclerosis. Dig Dis Sci 2008;53:1163-74.

5 Sallam H, McNearney TA, Chen JD. Systematic review: pathophysiology and management of gastrointestinal dysmotility in systemic sclerosis (scleroderma). Aliment Pharmacol Ther 2006;23:691-712.

6 Carette $S$, Lacourciere $Y$, Lavoie $S$, et al. Radionuclideesophageal transit in progressive systemic sclerosis. J Rheumat 1985;12:478-81.

7 Clements PJ, Becvar R, Drosos AA, et al. Assessment of gastrointestinal involvement. Clin Exp Rheumatol 2003;21(3. Suppl. 29):515-18.

8 Ebert EC. Gastric and enteric involvement in progressive systemic sclerosis. J Clin Gastroenterol 2008;42:5-12.

9 Herrick AL, Barlow JD, Bowden A, et al. Investigation of ananl function in patients with systemic sclerosis. Ann Rheum Dis 1996;55:370-74.

10 Kim KC, Park HJ, Lee SH, et al. Anorectal dysfunction in systemic sclerosis. J Korean Med Sci 1996;11:244-49.

11 Massone C, Milione L, Parodi A, et al. Anorectal involvement is frequent in limited systemic sclerosis. Acta Derm Venereol 2002;82:446-48.

12 Bellando Randone S, Guiducci S, Matucci Cerinic M. Patients subgroups and potential risk factors in systemic sclerosis: is there a possibility of an early diagnosis? Int J Clin Rheumatol 2010;5:555-64.

13 Avouac J, Fransen J, Walker UA, et al. Preliminary criteria for the very early diagnosis of systemic sclerosis: results of a Delphi Consensus Study from EULAR Scleroderma Trials and Research Group. Ann Rheum Dis 2011;70:476-81.

14 Conchillo JM, Smout AJ. Review article: intra-oesophageal impedance monitoring for the assessment of bolus transit and gastro-oesophageal reflux. Aliment Pharmacol Ther 2009;29:3-14.

15 Cutolo M, Pizzorni C, Meroni M, et al. The role of nailfold videocapillaroscopy in Raynaud's phenomenon monitoring and early diagnosis of systemic sclerosis. Reumatismo 2010;62:237-47.

16 Arndorfer RC, Stef JJ, Dodds WJ, et al. Improved infusion system for intraluminal esophageal manometry. Gastroenterology 1977;73:23-7.

17 Murray JA, Clouse RE, Conklin JL. Components of the standard oesophageal manometry. Neurogastroenterol Motil 2003;15:591-606.

18 Rao SSC, Azpiroz F, Diamant N, et al. Minimum standards of anorectal manometry. Neurogastroenterol Mot 2002;14:553-59.

19 Valentini G, Cuomo G, Abignano G, et al. Early systemic sclerosis: assessment of clinical and pre-clinical organ involvement in patients with different disease features. Rheumatology 2011;50:317-23.

20 Matucci Cerinic M, Bellando Randone S, Lepri G, et al. Very early versus early disease: the evolving definition of the many faces of Systemic Sclerosis. Ann Rheum Dis 2013;72:319-22.

21 Rattan S. The internal anal sphincter: regulation of smooth muscle tone and relaxation. Neurogastroenterol Motil 2005;15(Suppl. 1):50-9.

22 Ponge T, Bruley des Varannes S. Digestive involvement of scleroderma. Rev Prat 2002:52:1896-900. 